\title{
In silico Prioritization of some Tetrazole Chalcones for Anticonvulsant Activity
}

\author{
${ }^{*}$ Mohite P.B. ${ }^{1}$, Pawar D.D. ${ }^{1}$ \\ ${ }^{1}$ Department of Pharmaceutical Chemistry, MES's College of Pharmacy, Sonai, \\ Tal-Newasa, Dist-Ahmednagar, Maharashtra, India
}

\begin{abstract}
In silico predictions of molecules have quenched the thirst of drug discovery as it provides insight for the prioritization of the molecules for in vivo or in vitro pharmacological evaluations. Molecules for series PTC $_{1-7}$ and CPTC $_{1-7}$ were subjected for in silico biological activity predictions, partition coefficient predictions ( $\mathrm{pLog} \mathrm{P}$ ) and ADME predictions using PASS server, mol-inspiration software and PreADMET software respectively. This gave biological activity score (BAS), ADME predictions and LogP predictions ( $\mathrm{pLog} \mathrm{P}$ ) for anticonvulsant activity. The standard Log P required for anticonvulsant activities being +2.00 , molecules were also prioritized based on this pLogP criteria. In the protocol for BAS prediction, clinically used anticonvulsant agents were included and similarly followed for Log P predictions. The protocol was thus validated by comparing the correlation between pLog P and BAS. Molecules were also prioritized using the above protocol. The BAS score for selected the first five prioritized molecules was in the range of $0.30-0.61$, Memantine showed a BAS of 0.93 , which was used as standard in the prioritization protocol. The molecules $\mathrm{CPTC}_{3}$ showed a BAS of 0.61 as compared with Memantine with BAS of 0.93. PTC $\mathbf{P}_{3}, \mathbf{P T C}_{2}, \mathbf{P T C}_{1}, \mathbf{C P T C}_{2}$, $\mathrm{CPTC}_{3}$, \& CPTC $_{4}$ can serve is an in silico lead and outcome of in silico virtual screening for BAS predictions with ADME property \& pLog P prioritization. Thus molecules were prioritized for anticonvulsant activity.
\end{abstract}

Keywords: chalcones, In silico, BAS,PASS.

\section{Introduction}

An in silico study for the prioritization of 3-substituted phenyl-1-(5-phenyl-1H-tetrazol-1 yl)prop-2-

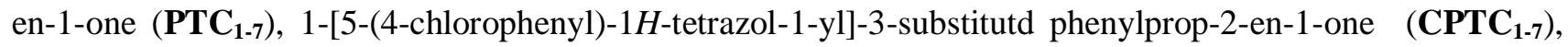
derivatives was performed for Biological activity score (BAS) prediction for anticonvulsant activity, Predicted LogP Predictions (PLogP), and ADME Predictions Using Pass Server For Biological activity predictions (http//195.178.207.233/ PASS/BAS.html) ${ }^{1}$, Mol inspiration Server (www.molinspiraion.com) ${ }^{2}$ and PreADMET Server (www.bmdrc.com/04_product/01_preadme.asp ) ${ }^{3}$ respectively. In present work the Nheterocyclic chalcones were prioritized for anticonvulsant activity.

Mohite P.B. et al /International Journal of PharmTech Research, 2019,12(2): 155-161.

DOI: http://dx.doi.org/10.20902/IJPTR.2019.120210 


\section{PASS Server Biological Activity Predictions ${ }^{1}$}

PASS Inlet predicts biological activity spectrum (783 pharmacological effects, mechanisms of action, specific toxicity) on the basis of structural formula of the compound. In the "Prediction Results" one obtains the total number of chemical descriptions of compound, and the number of descriptors which are new comparing to the descriptors in 30,900 compounds from the PASS training set. The compounds are considered equivalent in PASS if they have the same molecular formulae. The result of prediction is presented as the list of activities with appropriate $\mathrm{Pa}$ and $\mathrm{Pi}$, sorted in descending order of the difference $(\mathrm{Pa}-\mathrm{Pi})>0$ ( $\mathrm{Pa}$ values are given in this research paper which are the Biological activity scores. Biological activity spectrum with $\mathrm{Pa}$ value is termed as biological activity score. Biological Activity Spectrum of a compound represents the complex of pharmacological effects, physiological \& biochemical mechanisms of action, specific toxicity (mutagenicity, carcinogenicity, teratogenicity \& embryo toxicity) which can be revealed in compound's interaction with biological system. Biological activity spectrum with $\mathrm{Pa}$ values is termed as biological activity score (BAS). Biological Activity Spectrum describes the intrinsic properties of the compound depended on it's the structural particularities. They may be revealed in experiment under any conditions (dosage, route of administration, biological object, age, sex, etc.). BAS for anticonvulsant activity were determined for some as chalcones of tetrazole molecules in this research paper.

\section{2. pLogP predictions ${ }^{5}$}

It is the ratio of the compound's organic (oil)-to-aqueous phase concentrations.

\section{Partition Coefficient $(\mathbf{P})=[$ Organic $] /[$ Aqueous $]$}

Log $P$ of compound should be greater than 2.00 for compound to cross of blood brain barrier. Because central nervous system (CNS)-active compounds must pass across it and CNS-inactive compounds mustn't pass across it in order to avoid of CNS side effects. Hydrophobicity of a compound (as measured by its distribution coefficient) is a major determinant of how drug-like it is. More specifically, in order for a drug to be orally absorbed, it normally must first pass through lipid bilayers in the intestinal epithelium (a process known as transcellular transport). LogP is used in QSAR studies and rational drug design as a measure of molecular hydrophobicity.

\section{Mol inspiration software}

Mol inspiration is versatile cheminformatics software tool supporting molecule manipulation and processing, including SMILES and SDfile conversion, normalization of molecules, generation of tautomers, molecule fragmentation, calculation of various molecular properties needed in QSAR, molecular modelling and drug design, high quality molecule depiction, molecular database tools supporting substructure search or similarity and pharmacophore similarity search. Mol inspiration is independent platform and may be run on any PC, Mac, UNIX or LINUX machine. It offers contract research in all areas of cheminformatics, including: 1. calculation of molecular properties for large molecular databases 2. QSAR and structure-activity analysis. 3. Development of activity models for (nearly) any required target, efficient fragment-based virtual screening. 4. Design of targeted combinatorial libraries. 5. Diversity selection from large molecular collections.

\section{ADME Predictions $6,7,10$}

ADME means absorption, distribution, metabolism and excretion, which are major parts of pharmacokinetics. Statistics reports show that many of drug candidates are failed during clinical tests because of the problems related to ADME. Hence In silico ADME properties are used to prioritize molecules. The in silico ADME parameters and their ranges used for prioritization are mentioned under each ADME property.

\section{a. Caco2 Cell Permeability ${ }^{6,7}$}

Caco-2 cells tool for intestinal cell function and differentiation. Caco2 cells are derived from human colon Adenocarcinoma and possess multiple drug transport pathways through the intestinal epithelium. For prediction of Caco- 2 cell permeability in PreADMET, Molecules are solvated in silico at $\mathrm{pH} 7.4$, because Caco-2 cell permeability measured at about $\mathrm{pH}$ 7.4. Caco2 cells are used to determine the apparent 
permeability values of compounds. The ranges of Caco2 cell permeability used for in silico prioritization are shown in Table-1.

Table .1. Showing ranges of Caco2 cell permeability prediction

\begin{tabular}{|c|c|}
\hline Classification & PCaco-2 $(\mathrm{nm} / \mathrm{sec})$ \\
\hline Low permeability & less than 4 \\
\hline Middle permeability & $4 \sim 70$ \\
\hline High permeability & more than 70 \\
\hline
\end{tabular}

\section{MDCK cell permeability ${ }^{6,10}$}

MDCK cell means Madin-Darby canine kidney cell. Experimental and computational screening models for the prediction of intestinal drug absorption. MDCK cells are used to determine out the apparent permeability values of compounds. The ranges of MDCK cell permeability used for in silico prioritization of molecule are shown in Table-2.

Table . 2. Showing ranges of MDCK cell permeability predictions

\begin{tabular}{|l|l|}
\hline Classification & PMDCK $(\mathrm{nm} / \mathrm{sec})$ \\
\hline Low permeability & less than 25 \\
\hline Middle permeability & $25 \sim 500$ \\
\hline High permeability & more than 500 \\
\hline
\end{tabular}

\section{Human Intestinal Absorption (HIA)}

Predicting human intestinal absorption of drugs is very important for identify potential drug candidate. PreADMET can predict percent human intestinal absorption (\%HIA). Human intestinal absorption data are the sum of bioavailability and absorption evaluated from ratio of excretion or cumulative excretion in urine or bile. The ranges of HIA predictions are shown in Table-3.

Table .3. Showing ranges of HIA predictions

\begin{tabular}{|l|l|}
\hline Classification & HIA (Human Intestinal Absorption) \\
\hline Poorly absorbed compounds & $0 \sim 20 \%$ \\
\hline Moderately absorbed compounds & $20 \sim 70 \%$ \\
\hline Well absorbed compounds & $70-100 \%$ \\
\hline
\end{tabular}

\section{Blood Brain Barrier Penetration}

Blood-Brain Barrier (BBB) penetration is represented as $\mathrm{BB}=[$ Brain $] /[$ Blood $]$, where $[$ Brain $]$ and [Blood] are the steady-state concentration of radio labeled compounds in brain and peripheral blood. Predicting BBB penetration means predicting whether compounds pass across the blood-brain barrier. This is crucial in pharmaceutical sphere because CNS-active compounds must pass across it and CNS-inactive compounds mustn't pass across it in order to avoid of CNS side effects. PreADMET can predict in vivo data on rates for BBB penetration. The ranges of blood brain barrier predictions are shown in Table- 4 .

Table .4. Showing ranges of $\mathrm{BBB}$ predictions

\begin{tabular}{|l|l|}
\hline Classification & BB (Cbrain/Cblood) \\
\hline CNS - Active compounds & $(+)$ more than 1.0 \\
\hline CNS- Inactive compounds & (- ) less than 1.0 \\
\hline
\end{tabular}




\section{Plasma Protein Binding (PBB)}

Generally, only the unbound drug is available for diffusion or transport across cell membranes, and also for interaction with a pharmacological target. As a result, a degree of plasma protein binding of a drug influences not only on the drug's action but also its disposition and efficacy. PreADMET can predict percent drug bound in plasma protein as in vitro data on human. The ranges of PBB used for in siico prioritization are shown in Table-5.

\section{Table .5. Showing ranges of PBB predictions}

\begin{tabular}{|l|l|}
\hline Classification & Plasma Protein Binding (\%PPB) \\
\hline Chemicals strongly bound & more than $90 \%$ \\
\hline Chemicals weakly bound & less than $90 \%$ \\
\hline
\end{tabular}

Our main objectives were to in silico prioritize molecules for actual synthesis and evaluation based upon BAS, pLogP and PreADMET predictions. Correlation coefficients were obtained for correlation between BAS and pLog P, to find a correlation between pLog P and BAS predicted by the Server.

\section{Materials and Methods}

\section{In silico screening ${ }^{13-15}$}

Chemdraw 8.0 was used to convert 2-D chemskech files into 3-D Mol Files. Further these were uploaded into the server to obtain BAS activity Predictions, pLoP values and ADME predictions respectively.

a) BAS

Mol files of series of 3-substituted phenyl-1-(5-phenyl-1H-tetrazol-1 yl)prop-2-en-1-one (PTC 1 -7), 1[5-(4-chlorophenyl)-1H-tetrazol-1-yl]-3-substitutd phenylprop-2-en-1-one (CPTC 1-7 , were subjected to predict BAS usin PASS server. The structures of the compounds are shown in figure no. 1. These values are shown in Table No 6.

\section{b) Log P predictions pLogP}

Mol files of series of molecules from PTC $_{1-7}$ and $\mathbf{C P T C}_{1-7}$ series were subjected to predict $\log \mathrm{P}$ values (pLog P) using Molinspiration software available on line on www. Molinspiration.com an attempt was made to correlate the BAS with pLog P and its graph is shown in figure no 2 .

\section{c) ADME predictions}

Mole files of series of molecules from series of molecule (PTC1-7 and CPTC1-7) were subjected to predict ADME properties mentioned earlier. These values are shown in Table no-6.

\section{d) Prioritization of the molecules ${ }^{12}$}

The molecules having acceptable BAS, LogP and ADME properties were prioritized for actual synthesis and biological evaluation as anticonvulsant agents. 
<smiles>[R]C=CC(=O)n1nnnc1-c1ccc([R])cc1</smiles>

$\mathbf{R}^{1}=\mathbf{H}=\mathbf{P T C}_{\mathbf{1 - 7}}$

$\mathbf{R}^{1}=\mathrm{Cl}=\mathrm{CPTC}_{1-7}$

$\mathrm{R}=\mathrm{C}_{6} \mathrm{H}_{5}, 4-\mathrm{ClC}_{6} \mathrm{H}_{4}, 4-\mathrm{BrC}_{6} \mathrm{H}_{4}, 4-\mathrm{CH}_{3} \mathrm{C}_{6} \mathrm{H}_{4}, 4-\mathrm{OCH}_{3} \mathrm{C}_{6} \mathrm{H}_{4}, 4-\mathrm{NO}_{2} \mathrm{C}_{6} \mathrm{H}_{4}, 4-\mathrm{N}\left(\mathrm{CH}_{3}\right)_{2} \mathrm{C}_{6} \mathrm{H}_{4}$.

Figure 2: Structures of different N-Heterocyclic Chalcones

Table 6: showing prioritization of molecules

\begin{tabular}{|c|c|c|c|c|c|c|c|}
\hline \multirow{2}{*}{ Comp. } & \multirow{2}{*}{ BAS } & \multirow{2}{*}{ plogP } & \multicolumn{5}{|c|}{ ADME prediction } \\
\cline { 4 - 8 } & & & Caco 2 cell & MDCK cell & HIA & PPB & BBB \\
\hline PTC $_{1}$ & 0.45 & 3.66 & 21.00 & -2.96 & 98.24 & 100 & 2.76 \\
\hline PTC $_{2}$ & 0.57 & 4.34 & 21.82 & -2.96 & 97.64 & 99.54 & 2.27 \\
\hline PTC $_{3}$ & 0.50 & 4.47 & 21.66 & -3.01 & 97.42 & 100 & 2.42 \\
\hline PTC $_{4}$ & 0.43 & 4.11 & 20.83 & -2.88 & 98.11 & 100 & 3.13 \\
\hline PTC $_{5}$ & 0.32 & 3.76 & 22.08 & -2.89 & 98.10 & 100 & 1.58 \\
\hline PTC $_{6}$ & 0.30 & 3.18 & 21.11 & -3.21 & 96.80 & 94.67 & 0.50 \\
\hline PTC $_{7}$ & 0.30 & 3.72 & 21.79 & -3.17 & 98.76 & 97.28 & 1.37 \\
\hline PPTC $_{1}$ & 0.57 & 4.34 & 21.64 & -3.01 & 97.64 & 99.68 & 2.22 \\
\hline PPTC $_{2}$ & 0.56 & 5.01 & 22.35 & -2.95 & 97.39 & 100 & 1.00 \\
\hline PPTC $_{3}$ & 0.61 & 5.15 & 22.18 & -2.91 & 97.45 & 100 & 1.04 \\
\hline CPTC $_{4}$ & 0.52 & 4.79 & 21.99 & -2.95 & 97.55 & 97.78 & 2.19 \\
\hline CPTC $_{5}$ & 0.41 & 4.43 & 20.79 & -3.26 & 96.68 & 100 & 0.17 \\
\hline CPTC $_{6}$ & 0.39 & 3.86 & 24.00 & -3.02 & 97.55 & 93.34 & 1.15 \\
\hline CPTC $_{7}$ & 0.39 & 4.39 & 22.23 & -3.22 & 98.16 & 96.84 & 1.31 \\
\hline
\end{tabular}

Prioritization of molecules

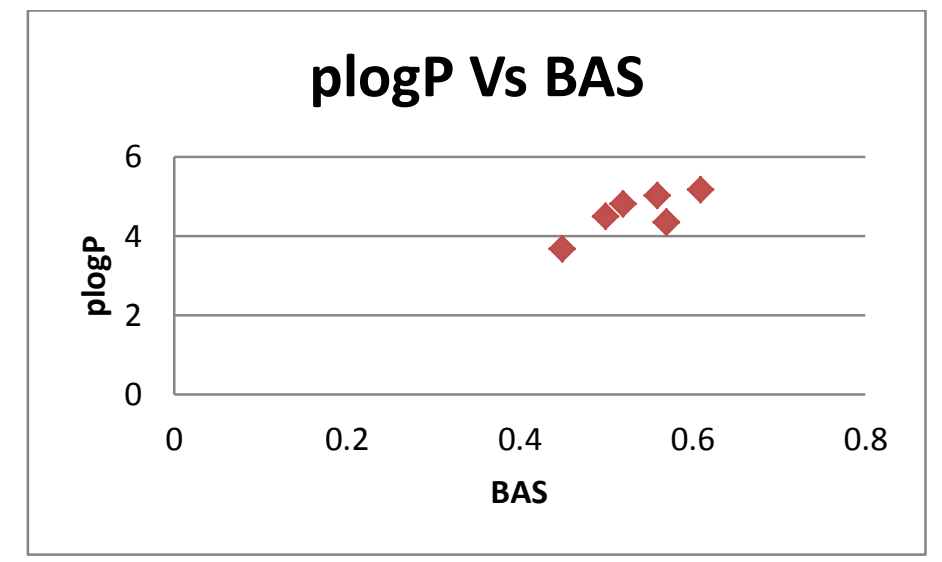

Figure 2: showing the correlation between plogP and BAS 


\section{Result and Discussion}

\section{BAS}

$\mathrm{Pa}$ and $\mathrm{Pi}$ are the estimates of probability for the compound to be active and inactive respectively for each type of activity from the biological activity spectrum. Their values vary from 0.000 to 1.000 . It is reasonably that only those types of activities may be revealed by the compound, where $\mathrm{Pa}>\mathrm{Pi}$ (only $\mathrm{Pa}$ is reported here) and so they are put into the biological activity spectrum. The $\mathrm{Pa}$ and $\mathrm{Pi}$ values obtained from the Pass server shall be utilized as follows: 1) If $\mathrm{Pa}>0.7$ the compound is very likely to reveal this activity in experiments, but in this case the chance of being the analogue of the known pharmaceutical agents for this compound is also high. 2) If $0.5<\mathrm{Pa}<0.7$ the compound is likely to reveal this activity in experiments, but this probability is less, and the compound is not so similar to the known pharmaceutical agents. 3) If $\mathrm{Pa}<0.5$ the compound is unlikely to reveal this activity in experiments, but if the presence of but this probability is less, and the compound is not so similar to the known pharmaceutical agents. 4) If $\mathrm{Pa}<0.5$ the compound is unlikely to reveal this activity in experiments, but if the presence of this activity is confirmed in the experiment the compound might be a New Chemical Entity. The BAS of selected molecules was found to be PTC $_{2}-0.57, \mathbf{P T C}_{3}-0.50$, PTC $_{1}-0.45, \mathbf{C P T C}_{3^{-}}-0.61, \mathbf{C P T C}_{2^{-}}-0.55, \mathbf{C P T C}_{4^{-}}$0.52, respectively. Hence these compounds have high $\mathrm{Pa}$ value for being prioritized for actual synthesis and evaluation for anticonvulsant activity.

\section{2.pLog $P$}

For anticonvulsant activity the pLog P should be greater than 2.00 , hence compounds above 2.00 pLog P were prioritized based on pLog P criteria. CNS active compounds must pass across it, in order to avoid of CNS side effects. The pLogP value of selected molecule was found to be $\mathbf{P T C}_{2^{-}}$4.34, PTC $\mathbf{3}^{-4.47}$ ,PTC $\mathbf{1}^{-3.66}$, CPTC $_{3}-5.15$, CPTC $_{2}-5.01$, CPTC $_{4}-4.79$,respectively. And comply for being prioritized as CNS active agents for anticonvulsant activity.

\section{Correlation between the Biological activity score and $\log P$}

In order to determine correlation between the BAS and pLogP, correlation coefficient was obtained for the BAS and pLogP, However it was found that the BAS and predicted Log P are not in any relation and the BAS is independent of pLog P predictions since the Coefficient of correlation between the pLog P and biological activity score was found to be 0.73 .

\section{ADME predictions}

ADME predictions based on in silico predictions of Caco2, MDCK, PBB, HIA, BBB etc. they are mentioned in Table no-6. The compound such as $\mathbf{P T C}_{\mathbf{2}}, \mathbf{P T C}_{\mathbf{3}}, \mathbf{P T C}_{\mathbf{1}}, \mathbf{C P T C}_{\mathbf{3}}, \mathbf{C P T C}_{\mathbf{2}}, \mathbf{C P T C}_{\mathbf{4}}$, lie in the range of in silico Caco2 cell, MDCK cell, HIA, PBB and BBB predictions and hence prioritized in silico for ADME properties.

\section{Conclusion}

BAS and pLogP were devoid of any correlations. Following the above protocol molecules from series $\mathbf{P T C}_{2}, \mathbf{P T C}_{3}, \mathbf{P T C}_{1}$, series viz. CPTC $2, \mathbf{C P T C}_{3}, \mathbf{C P T C}_{4}$, were prioritized with in silico technique, for synthesis and pharmacological screening as anticonvulsant agents. The molecules showed comparable score of 0.61-0.45 as anticonvulsant agents as compared with Memantine used as standard in the BAS prediction. These molecules can serves as leads for anticonvulsant activity and are outcome of in-silico screening for pLogP, ADME and BAS score prioritization.

\section{Acknowledgements}

The author is thankful to Principal MES's college of Pharmacy, Sonai, for their support and providing the research facilities. 


\section{References}

1. http//www.195.178.207.233/PASS/BAS.html

2. www.molinspiration.com

3. www.bmdrc.com/04_product/01_preadme.asp

4. Loscher W. New visions in the pharmacology of anticonvulsant. Europena Journal of Pharmacology.1998; 342(1):1-13, doi:10.1016/S0014-2999(97)01514-8.

5. Sachin S. Laddha and Satyendra P. Bhatnagar $13^{\text {rd }}$ international conference on synthetic organic chemistry, 2009;1-30 .

6. MDCK (Madin-Darby canine kidney) cells: A tool for membrane permeability screening. By J. D. Irvine and et.al, Journal of Pharmaceutical Sciences,1999; Vol. 88,No. 1.

7. Jibin $\mathrm{Li}$, Use of transporter knockdown caco-2 cells to investigate the in vitro efflux of statin drugs , Drug metabolism and description 2011; March 29-35,.

8. U.L. Narayan, A. G. Nerkar and C.S. Panda, Synthesis and screening of 6,6' methylene bis-2-phenyl3-[2'-aryl-thiazolidin-4-one]/[2'- aryloxazolidin-4-one'] Quinazolin-4-3H-ones for antibacterial and antiplatelet activities. International Journal of Chemical Sciences. 2006; 4:1-9.

9. U.L. Narayan, A. G. Nerkar and C.S. Panda, Synthesis and screening of 6,6' methylene bis-2-phenyl3-[2'-aryl-thiazolidin-4-one]/[2'- aryl-imidazolidin-4-one'] Quinazolin-4-3H-ones for antibacterial and antifungal activities. International Journal of Chemical Sciences. 2006; 4 ;93-100.

10. Charles B. Clayman, MD, American Medical Association Encyclopedia of Medicine, Medical Editor, Random House, New York, 1989.

11. S.Parasuraman, Prediction of activity spectra for substances, Journal of Pharmacology and Pharmacotherapeutics, 2011,2,1,52-53, doi:10.4103/0976-500X.77119.

12. K. S. Lade A. G. Nerkar, and S.D.Sawant In silico prioritization of some quinazolinone for anticonvulsant activity, Journal of Pharmacy Research, 2011;4(8),2800-2802.

13. Nerkar AG, Kudale SA, Joshi PP and Chikhale HU, in silico screening, synthesis and pharmacological evaluation of novel quinazolinones as nmda receptor inhibitors for anticonvulsant activity. International Journal of Pharmacy and Pharmaceutical Sciences. 2012, 4(3), 449-53.

14. Rajveer $\mathrm{C} \mathrm{H}$, Swarnalatha $\mathrm{C} \mathrm{H}$, Synthesis of 6-bromo-oxo quinazoline derivatives and their pharmacological activities, International Journal of Chemistry Research. 2010, 1(1), 1-4.

15. Loscher W, New visions in the pharmacology of anticonvulsion. European Journal of Pharmacology, 1998; 342(1):1-13, doi:10.1016/S0014-2999(97)01514-8. 